\title{
A Numerical Algebraic Geometry Approach To Regional Stability Analysis of Polynomial Systems
}

\author{
Frank Permenter ${ }^{1}$ and Charles Wampler ${ }^{2}$ and Russ Tedrake ${ }^{3}$
}

\begin{abstract}
We explore region of attraction (ROA) estimation for polynomial systems via the numerical solution of polynomial equations. Computing an optimal, stable sub-level set of a Lyapunov function is first posed as a polynomial optimization problem. Solutions to this optimization problem are found by solving a polynomial system of equations using techniques from numerical algebraic geometry. This system describes KKT points and singular points not satisfying a regularity condition. Though this system has exponentially many solutions, the proposed method trivially parallelizes and is practical for problems of moderate dimension and degree. In suitably generic settings, the method solves the underlying optimization problem to arbitrary precision, which could make it a useful tool for studying popular semidefinite programming based relaxations used in ROA analysis.
\end{abstract}

\section{INTRODUCTION}

Suppose an ordinary system of differential equations has an equilibrium point. A basic problem in nonlinear systems theory is estimating the region of attraction (ROA) of the equilibrium, which is defined as the set of initial conditions that converge to the equilibrium as time tends to infinity. It is common to inner approximate the region of attraction with the sub-level set of a Lyapunov function. If the time derivative of the Lyapunov function is negative definite in a sub-level set, then the sub-level set is contained by the ROA. It is therefore natural to ask what is the largest sub-level set for which this condition holds.

This problem can be cast as a non-linear programming problem which in general is computationally difficult. This problem remains hard if one only considers polynomial nonlinearity [1], but specialized solution techniques become available. In this paper, we describe a solution technique arising from the field of numerical algebraic geometry and demonstrate it is computationally feasible to numerically compute the optimal sub-level set for polynomial systems of reasonably low dimension (e.g. 1-6 states) in a reasonable amount of time (sub-second to 10 minutes). Unlike classical algorithms from algebraic geometry, the described solution technique does not rely on symbolic computation and trivially parallelizes.

Regional stability analysis of course goes beyond showing stability of a sub-level set of a Lyapunov function. Indeed, finding a Lyapunov function is in its own right a difficult

\footnotetext{
${ }^{1}$ F. Permenter and Russ Tedrake ${ }^{3}$ are with the Computer Science and Artificial Intelligence Laboratory (CSAIL), Massachusetts Institute of Technology, Cambridge, MA 02139. \{fperment, russt\}@mit.edu

${ }^{2}$ Charles Wampler is with General Motors Research and Development, Mail Code 480-106-224, 30500 Mound Road, Warren, MI 48090. Charles.W.Wampler@gm.com, www.nd.edu/ cwample1.
}

problem. Nevertheless, we feel the proposed method could be a useful computational tool for stability analysis.

\section{PRIOR WORK}

Many techniques exist for studying regional stability of polynomial dynamical systems. Perhaps the most common are methods based on sums of squares optimization [11], [10], [5], [14]. These methods allow one to certify emptiness of semi-algebraic sets by solving a hierarchy of semidefinite programs (SDPs). The optimal sub-level set problem we consider (and many others) can be cast in this framework. However, the size of the SDP required to exactly compute the optimal sub-level set is currently poorly understood, and the SDPs typically solved in practice only provably yield inner approximations. Other approximation techniques based on semidefinite programming include [8], which employs the theory of moments, and [6] which considers a general problem of bounding distance to a polynomial hypersurface. The quality of the bound given in [8] improves as the size of the SDP increases, but it is only known that the bound is asymptotically tight. The paper [6] presents testable necessary and sufficient conditions for tightness of its bound, but no quality measure for a loose bound is given. The proposed method can assess the quality of each of these SDP based approaches on specific instances of many systems studied in practice. This could perhaps lead to better understanding of these powerful techniques.

Another method based on quantifier elimination has been proposed in [4]. While quantifier elimination is a powerful theoretical tool, it typically scales to only very small problems in practice.

Finally, methods based on polynomial equation solving have been suggested. For example, [7] finds points of tangency between the level set of a Lyapunov function $V(x)$ and the vanishing hypersurface of its time derivative $\dot{V}(x)$ using Groebner bases techniques. This tangency constraint encodes local optimality of the polynomial optimization problem we will consider if one ignores regularity conditions. In addition, [7] assumes finitely many local optimizers and relies on generally expensive computer algebra routines. Interval arithmetic methods are considered in [15] and [17]. These methods employ numerical procedures for solving polynomial systems and are therefore similar in spirit to the technique we describe. They also extend to non-polynomial systems. However, these methods struggle in the presence of positive dimensional solution sets, an area that numerical algebraic geometry handles gracefully. 
Numerical algebraic geometry has found application in optimal control, for example [2]. Numerical algebraic geometry is also a powerful tool for solving problems in kinematics [16]. We offer, to our knowledge, its first application to regional stability analysis.

\section{BACKGROUND}

We begin our discussion by reviewing basic Lyapunov theory for regional stability. We then give a non-linear programming based description of provably stable sub-level sets of Lyapunov functions.

\section{A. Regional Stability Analysis}

To begin, call a function $V(x)$ a Lyapunov function iff it is differentiable and positive definite with compact and connected sub-level sets. Note that the connected condition is non-standard, but is employed to simplify presentation. One can use $V(x)$ to study regional stability of the system $\dot{x}=f(x)$ by applying the following standard result:

Theorem 1: Suppose $V(x)$ is a Lyapunov function and $\dot{V}(x)=\nabla_{x} V(x)^{T} f(x)$ is continuous. Define $\Omega_{\rho}$ to be the sub-level set $\{w: V(w) \leq \rho\}$ and $\operatorname{int}\left(\Omega_{\rho}\right)$ to be its interior. If

$$
\forall x \in \operatorname{int}\left(\Omega_{\rho}\right) \backslash\{0\} \quad \dot{V}(x)<0,
$$

then $\operatorname{int}\left(\Omega_{\rho}\right)$ is contained by the region of attraction.

Theorem 1 states if $\dot{V}(x)$ is strictly negative for all nonzero points in the interior of a $V(x)$ sub-level set, then all solutions to $\dot{x}=f(x)$ starting in the interior of the sub-level set converge to the origin. It is therefore natural to ask what is the largest sub-level for which condition (1) holds. If we assume $\dot{V}(x)$ is negative in a neighborhood of the origin, we can define the largest sub-level set using the optimal value of a constrained optimization problem. Consider the following result, whose proof is straightforward and hence omitted:

Theorem 2: Let $B(0, \epsilon)$ be an open $\epsilon$-ball centered at the origin and $\overline{B(0, \epsilon)}$ its closure. Let $V(x)$ be a Lyapunov function and suppose $\dot{V}(x)$ is negative for all $x \in \overline{B(0, \epsilon)}$ $\{0\}$. If $V^{*}$ is the optimal value of

$$
\begin{array}{cc}
\underset{x \in \mathbb{R}^{n}}{\operatorname{minimize}} & V(x) \\
\text { subject to } & \dot{V}(x)=0, \\
& x \notin B(0, \epsilon)
\end{array}
$$

then $\dot{V}(x)<0$ for all $x$ in the set

$$
\operatorname{int}\left(\Omega_{V^{*}}\right) \backslash\{0\},
$$

where $\Omega_{V^{*}}$ is the sub-level set:

$$
\left\{x: V(x) \leq V^{*}\right\} .
$$

Further, $\Omega_{V *}$ is the largest sub-level set with this property.

The optimal sublevel set $\Omega_{V *}$ of Theorem 2 can be found if one can solve optimization problem (2). Since $V(x)$ has compact sub-level sets and is continuous, it is easy to show that the optimal value of (2) is achieved if (2) is feasible. The optimal value is achieved by regular points only if they satisfy Karush-Kuhn-Tucker (KKT) optimality conditions:

$$
\begin{aligned}
\nabla_{x} V(x)+\lambda \nabla_{x} \dot{V}(x)+\zeta \nabla_{x}\left(\epsilon-x^{T} x\right) & =0 \\
\dot{V}(x) & =0 \\
x^{T} x-\epsilon & \geq 0 \\
\zeta\left(\epsilon-x^{T} x\right) & =0 \\
\zeta & \geq 0
\end{aligned}
$$

We can simplify these conditions by noting if $\dot{V}(x)=0$ then $\epsilon-x^{T} x \neq 0$ by assumed negativity of $\dot{V}(x)$ over $\overline{B(0, \epsilon)} \backslash\{0\}$. Thus, if $\dot{V}(x)=0$ the complementary slackness condition implies $\zeta=0$. Therefore, a point $x$ satisfies the KKT conditions only if

$$
\begin{aligned}
\dot{V}(x) & =0 \\
\nabla_{x} V(x)+\lambda \nabla_{x} \dot{V}(x) & =0
\end{aligned} .
$$

The KKT conditions are necessary unless $x$ is a singular point of the curve $\dot{V}(x)=0$, i.e. $x$ solves the system:

$$
\begin{aligned}
\nabla_{x} \dot{V}(x) & =0 \\
\dot{V}(x) & =0
\end{aligned} .
$$

Calling $S_{\text {sing }}$ the set of non-zero points solving (5) and $S_{k k t}$ the set of non-zero $x \in \mathbb{R}^{n}$ that for some $\lambda \in \mathbb{R}$ solve (4), we can express the optimal value of (2) as

$$
V^{*}=\inf \left\{V(x): x \in S_{k k t} \cup S_{\text {sing }}\right\} .
$$

In this paper, we will use numerical algebraic geometry to study $S_{k k t} \cup S_{\text {sing }}$ when (4) and (5) are polynomial. In particular, a method is presented for checking the cardinality of $S_{k k t} \cup S_{\text {sing }}$ and for enumerating its elements in the finite case. We also describe how to lower bound (6) if $S_{k k t}$ is infinite.

\section{B. Numerical Algebraic Geometry}

For a detailed treatment of numerical algebraic geometry one can see the book [12]. We briefly overview high level topics, and first note that methods in numerical algebraic geometry study polynomial systems over the complex numbers. Thus, additional care must be taken to reason about the behavior (i.e. existence, cardinality, etc) of real solutions.

Letting $z$ denote an indeterminate in $\mathbb{C}^{n}$, we begin with the following definition:

Definition 1: A solution $z_{0} \in \mathbb{C}^{n}$ to a polynomial system $g(z)$ is isolated if and only if no other solution to $g(z)$ exists in a neighborhood of $z_{0}$.

A basic operation in numerical algebraic geometry is enumerating isolated solutions via homotopy continuation. To find the isolated solutions of some system $g(z)$, one defines a homotopy that smoothly deforms a start system $h(z)$ with known solutions into $g(z)$. The homotopy and the start system $h(z)$ can be designed so that solutions of $h(z)$ trace smooth, continuous paths to all isolated solutions of $g(z)$ with probability one. Smoothness and continuity allow one to find the isolated solutions of $g(z)$ by tracking each path using predictor-corrector methods. The appeal of this approach is two fold: (1) all solutions can be performed in floating 
point arithmetic and (2) each solution path can be tracked in parallel.

All solutions to a polynomial system are isolated if and only if the system has finitely many solutions. To describe how numerical algebraic geometry applies to systems with infinitely many solutions, we first define the notion of irreducible algebraic sets and irreducible components of an algebraic set. An algebraic set, i.e. the solution set of a system of polynomial equations, is irreducible if it cannot be written as the union of two of its proper algebraic subsets. Irreducible components of an algebraic set $Q=\{z \in$ $\left.\mathbb{C}^{n}: g(z)=0\right\}$ are irreducible algebraic sets $Q_{i}$ for which $Q=\cup_{i} Q_{i}$.

Numerical algebraic geometry exploits the fact that a random hyperplane intersects an irreducible algebraic set of complementary dimension in a non-empty finite set of points. Thus, points on a $d$ dimensional irreducible component of the set $Q$ are isolated solutions of an augmented system constructed from $g(z)$ and $d$ random, independent linear equations. These points can be uncovered using homotopy continuation to construct a witness point superset $W^{d}$ for dimension $d$. The set $W^{d}$ contains points on each irreducible component of dimension $d$ and so called junk points on higher dimensional components that are artifacts of the continuation process. Junk points can be removed to enable sophisticated algorithms for studying $Q$, but are benign in the procedure we describe in this paper. If $z \in \mathbb{C}^{n}$, one can build sets $W^{d}$ for $d \in[0, \ldots, n]$ to construct a witness superset for $Q$. The witness superset $W$ contains the sets $W^{d}$ and the intersecting hyperplanes for each dimension.

Given a witness superset, one immediately knows the dimension of $Q$ as well as the isolated solutions of $g(z)$. The dimension is simply the largest $i$ for which $W^{i}$ is nonempty. The isolated solutions are contained in $W^{0}$. Thus, by constructing a witness superset, one can simultaneously acquire the isolated solutions and determine if other positive dimensional solution components exist.

Witness supersets can also be used to lower bound a function over the real points in $Q$ in a very special case. Suppose a function $w(z): \mathbb{C}^{n} \rightarrow \mathbb{C}$ mapping $\mathbb{R}^{n}$ to $\mathbb{R}$ is constant over each irreducible component of $Q$. A lower bound for $w(z)$ over $Q \cap \mathbb{R}^{n}$ is obtained by minimizing $w(z)$ over the set of points in $W$ for which $w(z)$ is real valued. This procedure produces a lower bound because the irreducible component containing the minimizing point in $W$ may not intersect $Q \cap \mathbb{R}^{n}$.

These crucial properties enable an algorithm for inner approximating the optimal sub-level set of $V(x)$ given witness supersets $W_{\text {sing }}$ and $W_{k k t}$ for the complex solution components to (4) and (5). We discuss this algorithm in the next section.

\section{APPROACH}

In this section we give an algorithm for finding a sub-level set of a Lyapunov function $V(x)$ contained by the ROA. Under mild conditions, this set is the largest sub-level set of $V(x)$ satisfying the stability condition (1). The algorithm is also equipped with testable sufficient conditions to verify optimality.

Our procedure is explicitly given in Algorithm 1. When systems (4) and (5) have finitely many complex solutions, the algorithm essentially enumerates the sets of real solutions $S_{k k t}$ and $S_{\text {sing }}$ to compute the optimal sub-level set described by (6). If the KKT system (4) has infinitely many solutions, the algorithm minimizes $V(x)$ over the complex solutions for which $V(x)$ is real valued and positive. This leads to a lower bound of (6).

We now describe key steps of the procedure. First the algorithm computes witness supersets $W_{k k t}$ and $W_{\text {sing. It }}$ then verifies from $W_{\text {sing }}$ that (5) has only isolated solutions. This implies $S_{\text {sing }}$ is a finite set, a necessary requirement for our algorithm to proceed (see Remark 1). Next the algorithm minimizes $V(x)$ over the union of three sets $X_{1}, X_{2}$ and $X_{3}$ constructed from $W_{k k t}$ and $W_{\text {sing }}$. Sets $X_{1}$ and $X_{2}$ contain the sets of non-zero, real, isolated points $x$ that solve (4) and (5):

$$
\begin{aligned}
& X_{1}=\left\{x: x \neq 0,(x, \lambda) \in W_{k k t}^{0} \cap \mathbb{R}^{n+1}\right\} \\
& X_{2}=\left\{x: x \neq 0, x \in W_{\text {sing }}^{0} \cap \mathbb{R}^{n}\right\}
\end{aligned}
$$

The set $X_{3}$ arises from the positive dimensional components of the solution set of (4) satisfying the following property:

- $\exists(x, \lambda)$ in the component s.t. $V(x)$ is real valued and positive.

We can generate a set of samples $X_{3}$ from these components using $W_{k k t}$. In particular:

$$
\begin{aligned}
X_{3}=\left\{x \in \mathbb{C}^{n}\right. & :(x, \lambda) \in W_{k k t}^{d}, \\
& d>0, V(x) \in \mathbb{R}, V(x)>0\}
\end{aligned}
$$

By applying the following theorem, we can minimize $V(x)$ over these components by minimizing over the set of samples $X_{3}$ :

Theorem 3: If $f(z)$ and $g(z)$ are polynomials mapping $\mathbb{C}^{n-1}$ to $\mathbb{C}$, then $f(z)$ is constant on each irreducible component of the algebraic set:

$$
Q=\left\{(z, \lambda) \in \mathbb{C}^{n}: g(z)=0, \nabla_{z} f(z)+\lambda \nabla_{z} g(z)=0\right\}
$$

Proof: Due to space constraints, we offer a brief sketch. First, apply Cauchy-Riemann equations and the defining equations of $Q$ to show the directional derivative of $f(z)$ vanishes along any path through $Q$, if $f(z)$ is viewed as a function of the real and imaginary parts of $(z, \lambda)$ and $Q$ is viewed as a subset of $\mathbb{R}^{2 n}$. The result then follows immediately from the fact irreducible components of $Q$, if $Q$ is viewed as a subset of $\mathbb{R}^{2 n}$, are piecewise smoothly, path connected [9].

Letting $\rho$ denote

$$
\rho=\min \left\{V(x): x \in X_{1} \cup X_{2} \cup X_{3}\right\}
$$

gives the inequality:

$$
0<\rho \leq \inf \left\{V(x): x \in S_{k k t} \cup S_{\text {sing }}\right\} .
$$

The first inequality arises by construction of $X_{i}$ and positive definiteness of $V(x)$ over $\mathbb{R}^{n}$. The second inequality arises 
because $X_{3}$ is constructed from points on components that may not contain real solutions. This inequality is tight, i.e. the calculated sub-level set is optimal, if $V(x)=\rho$ for some $x \in S_{k k t} \cup S_{\text {sing }}$. Obvious sufficient conditions for this are as follows:

1) $V(x)=\rho$ for some $x$ in $X_{3} \cap \mathbb{R}^{n}$.

2) $V(x)=\rho$ for some $x$ in $X_{1} \cup X_{2}$.

Remark 1: System (5) is required to have finitely many complex solutions since it has no analogue to Theorem 3 . In other words, we cannot use $W_{\text {sing }}$ to bound $V(x)$ on the complex solutions to (5) for which $V(x)$ is real valued if infinitely many complex solutions exist.

Remark 2: Witness supersets $W_{k k t}$ and $W_{\text {sing }}$ can be extracted from a witness superset $W$ for the polynomial system

$$
\begin{aligned}
\dot{V}(x) & =0 \\
\mu \nabla_{x} V(x)+\lambda \nabla_{x} \dot{V}(x) & =0 \\
a_{1} \mu+a_{2} \lambda+1 & =0
\end{aligned}
$$

where $\mu$ is a new indeterminate and $a_{1}, a_{2} \in \mathbb{R}$ are random fixed parameters. Construct $W_{k k t}^{d}$ from points in $W^{d}$ with $\mu \neq 0$. Take $W_{\text {sing }}^{d}$ to be all points in $W^{d}$ with $\mu=0$. In practice, solving this combined system can be computationally cheaper than solving systems (4) and (5) seperately. If the systems are solved seperately, a solver cannot exploit the fact that solutions of (5) are indeed solutions to (4) "at infinity."

\section{EXAMPLES}

We discuss a few examples to illustrate the procedure and its scaling properties.

\section{A. Example 1: Van der Pol Oscillator}

Consider the dynamics of a time reversed Van der Pol oscillator:

$$
f(x)=\left(\begin{array}{c}
-x_{2} \\
x_{2} x_{1}^{2}+x_{1}-x_{2}
\end{array}\right) .
$$

This system has a stable equilibrium at the origin. To estimate the region of attraction, we will identify a suitable Lyapunov function $V(x)$ and solve the polynomial optimization problem (2) by applying Algorithm 1.

To find a Lyapunov function, we first compute a linearization $\dot{x}=A x$ of (9) about the origin. Next, we solve the Lyapunov equation for some positive definite matrix $Q \in \mathbb{S}_{n}^{2}$ :

$$
A^{T} P+P A=-Q .
$$

Choosing $Q$ to be the identity gives

$$
P=\left(\begin{array}{cc}
\frac{3}{2} & -\frac{1}{2} \\
-\frac{1}{2} & 1
\end{array}\right) \text {. }
$$

Noting that $\dot{x}=A x$ is stable, we conclude that $\dot{V}(x)$ is negative in a neighborhood of the origin for $V(x)=x^{T} P x$.

Since $V(x)$ satisfies the conditions of Theorem 2 we estimate the ROA by solving the polynomial optimization problem (2). We do this by generating a witness superset $W$

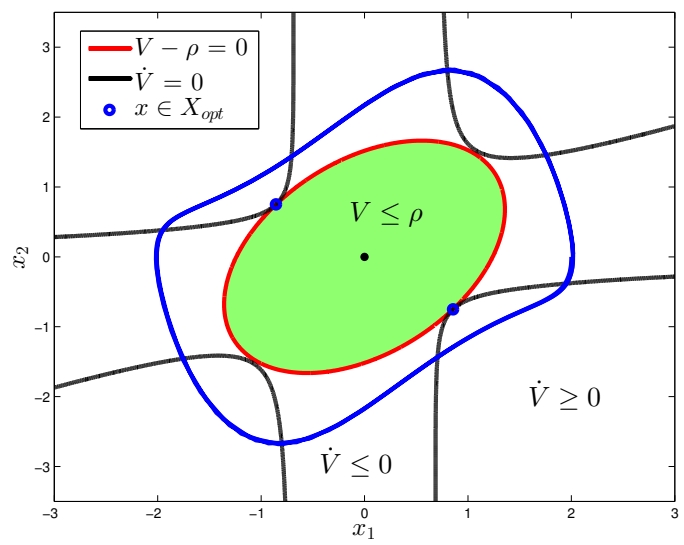

Fig. 1: Region of attraction estimate for the Van der Pol oscillator described in Section V-A. The set $X_{o p t}$ is the set of solutions to optimization problem (2) and defines the optimal sub-level set. The blue line indicates the boundary of the true ROA.

for the system (8). Introducing random real valued constants $a_{1}$ and $a_{2}$, this system takes the form:

$$
\begin{array}{r}
-x_{1}^{3} x_{2}+2 x_{1}^{2} x_{2}^{2}-x_{1}^{2}-x_{2}^{2}=0 \\
\mu\left(3 x_{1}-x_{2}\right)-\lambda x_{1}\left(-4 x_{2}^{2}+3 x_{1} x_{2}+2\right)=0 \\
-\mu\left(x_{1}-2 x_{2}\right)-\lambda\left(x_{1}^{3}-4 x_{2} x_{1}^{2}+2 x_{2}\right)=0 \\
a_{1} \mu+a_{2} \lambda-1=0
\end{array}
$$

From $W$ we construct witness supersets $W_{k k t}$ and $W_{\text {sing }}$ for systems (4) and (5). We calculate a stable sub-level set using the expression (7). $W_{k k t}$ and $W_{\text {sing }}$ reveal (4) and (5) have only isolated solutions satisfying $x \neq 0$. This implies the calculated sub-level set is optimal.

Constructing the witness superset $W$ for (10) took 0.6 seconds on an Intel(R) Core(TM) i7-2600K CPU @ 3.40GHz machine using the software package Bertini[3]. The calculated sub-level set is plotted in Figure 1, along with the set of $x$ that minimize (7), which we label $X_{\text {opt }}$. Note the geometric interpretation of optimality: the optimal $V(x)$ level set and curve $\dot{V}(x)=0$ are tangent.

\section{B. Applications to $N$ link manipulator}

We now illustrate the scaling properties of the algorithm by considering rigid body dynamics of a planar $N$ link manipulator pictured in Figure 2. The manipulator contains rotary joints at each link. A control torque is applied at all joints except for the base joint, hence this system is underactuated. For $N=2$, this system is sometimes called an acrobot [13]. We will study regional stability of the upright equilibrium point $\left(q_{i}=0, i=1, \ldots, N\right)$ when the system is under LQR control.

Each link is modeled as a rod with uniform mass. The base link has mass $1 \mathrm{~kg}$ and length $1 \mathrm{~m}$. All other links have mass $1.2 \mathrm{~kg}$ and and length $1.2 \mathrm{~m}$. Each joint has a viscous damping constant equal to $\frac{1}{10} N \cdot m \cdot s$.

We let $x=(q, \dot{q}) \in \mathbb{R}^{2 n}$ and let $u \in \mathbb{R}^{N-1}$ denote the control torques. We stabilize with an $L Q R$ controller calculated using dynamics linearized about the upright. Defining $I_{n}$ to be the identity matrix in $\mathbb{R}^{n \times n}$, we set $Q$ and $R$ equal 


\begin{tabular}{cccc}
$\mathrm{N}$ & Paths & $\sum_{i}\left|W^{i}\right|$ & Solve Time (s) \\
\hline 4 & 106633 & 12925 & 10600 \\
3 & 11945 & 1415 & 280 \\
2 & 1228 & 148 & 8.6
\end{tabular}

TABLE I: Metrics for the $N$-Link manipulator of Section V-B for different values of $N$. Shown are total paths tracked by Bertini [3] in the construction of witness point supersets $W^{i}$ for system (8). The total number of points in the $W^{i}$ is denoted $\sum_{i}\left|W^{i}\right|$. System (8) has one linear and $2 N+1$ quartic equations in $2 N+2$ variables. Computation was performed on an Intel(R) Core(TM) i7-2600K CPU @ 3.40GHz machine using 8 execution threads.

to

$$
Q=\left(\begin{array}{cc}
10 I_{n} & 0 \\
0 & I_{n}
\end{array}\right) \quad R=\left(\begin{array}{l}
I_{n-1} \\
\end{array}\right.
$$

We set $V(x)$ to be the LQR cost-to-go and Taylor expand the closed loop dynamics to 3rd order. We then construct the polynomial system (8), which contains $2 N+1$ quartic equations and one linear equation in $2 N+2$ unknowns. We calculate a witness superset $W$ for (8) and from it construct witness supersets $W_{k k t}$ and $W_{\text {sing }}$.

We consider cases for $N=2,3,4$. In all cases, the minimizing $x$ for (7) is real valued. We conclude the sublevel set defined by (7) is optimal for each choice of $N$. Computational summaries for witness superset calculations are given in Table I.

For the $N=2$ case, we plot slices of the calculated sublevel set in Figure 3. Taking $S$ to be to cost-to-go matrix and $T=S^{-1 / 2}$, we apply a change of coordinates $x=T w$ so that the Lyapunov function $V(T w)$ has circular sub-level sets in $w$. This is strictly for aesthetic purposes. We plot two slices of the ROA in these coordinates by fixing $w_{1}$ and $w_{3}$ (resp. $w_{2}$ and $w_{4}$ ) to the values given by $w=T x_{\text {opt }}$, where $x_{o p t}$ is the minimizer of the expression given by (7).

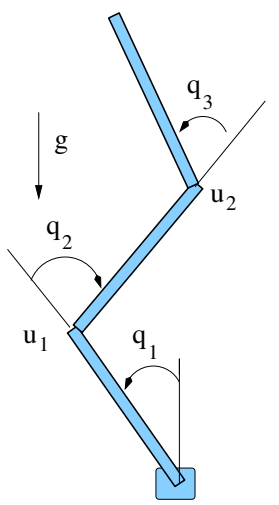

Fig. 2: The $N$-link planar manipulator considered in Section VB for $N=3$. Control torques $u_{1}$ and $u_{2}$ are applied to joints $q_{2}$ and $q_{3}$ respectively. System parameters for examples considered are given in Section V-B.

\section{DiscusSiON}

In each example we considered, $\dot{V}(x)$ had no non-zero singular points, i.e. system (5) had no non-zero solutions.
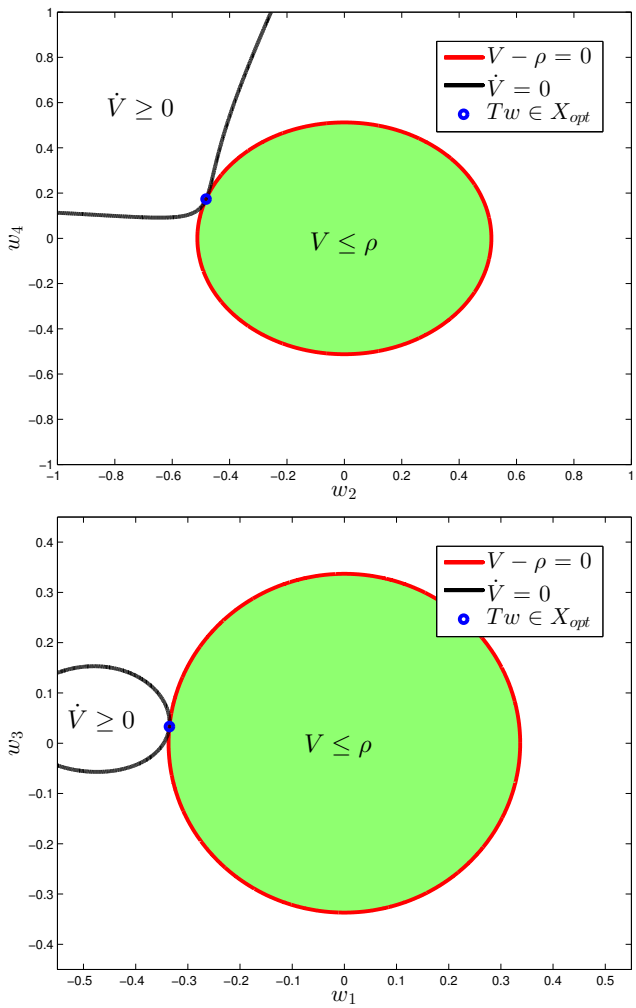

Fig. 3: $N$-Link Example. Slices of an ROA estimate found for a 2-link planar manipulator discussed in Section V-B. For plotting purposes, we have applied a change of coordinates $x=T w$ so that $V(T w)$ has circular level sets in $w$. The level set plotted is for $\rho=0.188$.

Moreover, the only solutions to the KKT system (4) satisfying $x \neq 0$ were isolated. This is perhaps not surprising, and suggests that heuristically searching for just the isolated solutions of the KKT system would be useful in practice. This simplication could yield significant computational savings.

If we were to adopt an approach of only finding isolated solutions, we could also apply coefficient-parameter homotopy theory to efficiently update the optimal sub-level set after changes to the Lyapunov function or system dynamics are made (perhaps an order of magnitude reduction in solution paths). Such an approach would perhaps be useful in controller design or in the search of Lyapunov functions.

\section{CONCLUSION}

In summary, we have proposed a method for region of attraction estimation based on numerical algebraic geometry and quantified its computational requirements. Under mild conditions, the proposed technique can find the optimal sublevel set to desired precision. This could make it useful for studying existing approximation algorithms for ROA estimation that sacrifice optimality for some other feature (e.g. speed). In addition, the algorithm recovers the optimal solution of (2) as opposed to just the optimal value. In contrast, recovery of the optimal solution in sums of squares methods, for example, is not always possible. It would be interesting to explore how optimal solutions can be used to 
Input: A polynomial Lyapunov function $V(x)$ and polynomial dynamical system $f(x)$ with equilibrium at the origin

Output: A parameter $\rho$ for which $\{x: V(x) \leq \rho\}$ is asymptotically stable.

\#Compute $\dot{V}(x)$, ensure it is negative around origin $\dot{V}(x)=\nabla_{x} V(x)^{T} f(x)$

unless $\nabla \dot{V}(0)=0$ and $\nabla^{2} \dot{V}(0) \prec 0$ return 0

\#Compute witness superset for polynomial system (8) $W=W$ itnessSuperSet $(V(x), \dot{V}(x))$

\#Split into witness supersets for (5) and (4). See Remark 2.

$\left[W_{k k t}, W_{\text {sing }}\right]=$ SplitWitnessSuperSet $(W)$

\#Require finitely many solutions to (5). See Remark 1. unless $\left|W_{\text {sing }}^{i}\right|==0 \forall i>0$ return 0

\#Build sets from Section IV and minimize $V(x)$

$\left[X_{1}, X_{2}, X_{3}\right]=\operatorname{BuildX} \operatorname{Set}\left(W_{k k t}, W_{\text {sing }}\right)$

$X=X_{1} \cup X_{2} \cup X_{3}$

$\rho=\min _{x \in X} V(x)$

\section{return $\rho$}

Algorithm 1: Computes a stable sub-level set of a Lyapunov function.

update Lyapunov functions, since they represent the precise points where the stability condition (1) fails.

\section{REFERENCES}

[1] A. A. Ahmadi, A. Majumdar, and R. Tedrake. Complexity of ten decision problems in continuous time dynamical systems. In To appear in ACC 2013.

[2] D. J. Bates, I.A. Fotiou, and Ph. Rostalski. A numerical algebraic geometry approach to nonlinear constrained optimal control. In IEEE Conference on Decision and Control, New Orleans, LA, dec 2007.

[3] D. J. Bates, J.D. Hauenstein, A.J. Sommese, and C.W. Wampler. Bertini: Software for numerical algebraic geometry. Available at http://www.nd.edu/ sommese/bertini.
[4] H. Burchardt and S. Ratschan. Estimating the region of attraction of ordinary differential equations by quantified constraint solving. In Proceedings of the 3rd WSEAS/IASME International Conference on Dynamical Systems and Control, CONTROL'07, pages 241-246, Stevens Point, Wisconsin, USA, 2007. World Scientific and Engineering Academy and Society (WSEAS).

[5] G. Chesi. Domain of Attraction: Analysis and Control via SOS Programming. Springer, 2011.

[6] G. Chesi, A. Tesi, A. Vicino, and R. Genesio. On convexification of some minimum distance problems. In European Control Conf, 1999.

[7] K. Forsman. Applications of Groebner bases to nonlinear systems. In In Proc. First European Control Conf, pages 164-169. Hermes, 1991.

[8] O. Hachicho and B. Tibken. Estimating domains of attraction of a class of nonlinear dynamical systems with LMI methods based on the theory of moments. In Decision and Control, 2002, Proceedings of the 41st IEEE Conference on, volume 3, pages 3150-3155. IEEE, 2002.

[9] M. Laurent. Sums of squares, moment matrices and optimization over polynomials. Emerging Applications of Algebraic Geometry, pages 157-270, 2009.

[10] A. Papachristodoulou and S. Prajna. On the construction of Lyapunoy functions using the sum of squares decomposition. In Proceedings of the 41st IEEE Conference on Decision and Control, Dec 2002.

[11] P. A. Parrilo. Structured Semidefinite Programs and Semialgebraic Geometry Methods in Robustness and Optimization. $\mathrm{PhD}$ thesis, California Institute of Technology, Pasadena, CA, 2000.

[12] A.J. Sommese and C.W. Wampler. The Numerical Solution Of Systems of Polynomials Arising in Engineering and Science. World Scientific Publishing, Hackensack, NJ, 2005.

[13] M. Spong. The swingup control problem for the acrobot. IEEE Control Systems Magazine, 15(1):49-55, February 1995.

[14] B. Tibken. Estimation of the domain of attraction for polynomial systems via LMIs. In Decision and Control, 2000. Proceedings of the 39th IEEE Conference on, volume 4, pages 3860-3864. IEEE, 2000.

[15] B. Tibken and O. Hachicho. Estimation of the domain of attraction for polynomial systems using multidimensional grids. In Proceedings of the IEEE Conference on Decision and Control, feb. 2000.

[16] C.W. Wampler and A.J. Sommese. Numerical Algebraic Geometry and Algebraic Kinematics. Acta Numerica, 20:469-567, 2011.

[17] S. Warthenpfuhl, B. Tibken, and S. Mayer. An interval arithmetic approach for the estimation of the domain of attraction. In ComputerAided Control System Design (CACSD), 2010 IEEE International Symposium on, pages 1999 -2004, sept. 2010. 